\title{
DUS Characterization of Bottle Gourd [Lagenaria siceraria (Mol.) Standl.] Genotypes
}

\author{
Gandamala Raghu*", Arun Kumar Chaurasia, K. Rohan Reddy, \\ Bineeta M. Bara and N. Nithyananda
}

Department of Genetics and Plant Breeding, Naini Agricultural Institute, Sam Higginbottom
University of Agriculture Technology and Sciences, Allahabad, U.P. India

*Corresponding author

\section{A B S T R A C T}

\begin{tabular}{|l|}
\hline K e y w o r d s \\
$\begin{array}{l}\text { Characterization, DUS } \\
\text { guidelines, PPV and FRA } \\
\text { guidelines, Bottle gourd, } \\
\text { Bottle gourd genotypes }\end{array}$ \\
\hline Article Info \\
\hline $\begin{array}{l}\text { Accepted: } \\
\text { 06 September } 2018 \\
\text { Available Online: } \\
\text { 10 October } 2018\end{array}$ \\
\hline
\end{tabular}

The Present study was carried out for characterization of Bottle gourd genotypes based on DUS guidelines. Four Bottle gourd genotypes were characterized based on the DUS guidelines given by PPV and FRA. A total of 31 characters given in DUS guidelines were used for characterization. The genotypes for study were obtained from Directorate of Research, Sam Higginbottom University of Agriculture Technology and Sciences, Allahabad, U.P. Among the 31 characters studied 18 characters were visually examined and 13 characters were measured. The fruit shape in longitudinal section was expressed as cylindrical (Desi Lauki-404), round (Desi Lauki Gol-417), elongate curved (Desi Lauki398) and (Lauki-426). The fruit skin colour has been grouped as dark green (Desi lauki398) and light green (Desi Lauki-404, Desi Lauki Gol-417, Lauki-426). There was no change in the expression of characters of the respective genotypes for a period of two consecutive years. In the present study the main focus was on characterization of the 4 bottle gourd genotypes based on DUS guidelines, tests for Distinctiveness, Uniformity and Stability were not part of the present study.

\section{Introduction}

The "Protection of Plant Varieties and Farmer's Rights Act" (PPV\&FR Act, 2001) was passed by the Government of India with the main objective of providing an effective system of protection against unlawful commercial exploitation of new plant varieties, the rights of farmers and plant breeders and to encourage the development of new varieties of plants. Genotypes of different crops are still unreleased as a commercial variety. Such genotypes can be characterized based on the DUS guidelines given by the PPV\&FRA for different crops. These set of guidelines help in the characterization of different genotypes based on morphology. There are mainly two categories of characterization that is based on visually observed characters and measurable characters. The genotypes are compared with different standard reference varieties with regard to their performance. Each and every character comprises of a reference variety by which the genotypes can be compared and categorized. The present study was carried out 
with the objective to 'characterize bottle gourd genotypes based on DUS guidelines'.

\section{Materials and Methods}

The study material comprised of genetically pure seed of 4 genotypes of Bottle gourd, viz. Desi Lauki-404, Desi Lauki-398, Desi Lauki Gol-417 and Lauki-426. The seeds of all the genotypes were sown with five rows of $6.4 \mathrm{~m}$ length keeping a row to row and plant to plant spacing of $4.0 \mathrm{~m}$ and $0.80 \mathrm{~m}$ respectively in Randomized Block Design and replicated thrice. The experiments were carried out at Field Experiment station, Sam Higginbottom University of Agriculture Technology and Sciences, Allahabad (Uttar Pradesh). For two consecutive years from kharif-2016 to 2017.

All genotypes under study were evaluated for 31 DUS characters at specified stage of crop growth period when the characters under study exhibited full expression following the guidelines of Srivatava et al., (2001). The observations for characterization of Bottle gourd genotypes based on DUS guidelines were made on plants randomly selected from each replication. The observations for few characters were made on group of plants or part of plants. For few characters only a single observation was recorded from a group of plants. All observations on leaf were recorded on fully developed leaves, preferably between $15^{\text {th }}$ and $20^{\text {th }}$ nodes. Fruit length and width was recorded at marketable maturity of the fruit. Observations on the ovary were recorded on the day of anthesis. All the observations on the seeds were made on fully developed, matured and dry seeds, after washing and drying.

\section{Results and Discussion}

Among the 4 bottle gourd genotypes considerable variation was observed for all important characters. The states of expression of particular traits of Bottle gourd genotypes are presented in Table 1, 2, 3, 4 and 5 respectively. In the present study all the 4 genotypes under study had andro monecious sex expression. The leaf shape of the genotypes was found to be Orbicular (Desi Lauki-404, Desi Lauki gol-417, Lauki-426) and Obovate (Lauki-398) (Table 1). The ovary length was expressed as short (Desi Lauki-398 and Desi Lauki Gol-417) and medium (Desi Lauki-404 and Lauki-426) (Table 6 and 7). On the basis of fruit shape the Bottle gourd genotypes have been grouped in to cylindrical (Desi Lauki-404), round (Desi Lauki Gol417), elongate curved (Desi Lauki-398 and Lauki-426) (Table 3). On the basis of fruit length the Bottle gourd genotypes have been grouped in to short (Desi Lauki Gol-417), medium (Desi Lauki-398), long (Desi Lauki404 and Lauki-426) (Table 6 and 7). On the basis of fruit skin colour the Bottle gourd genotypes have been grouped in to light green (Desi Lauki-404, Desi Lauki Gol-417, Lauki426) and Dark green (Desi Lauki-398) (Table 4) The studies of Sivaraj et al., (2005), Miadenovic et al., (2012), Mladenovic et al., (2011) described the morphological characterization of bottle gourd genotypes. Earlier morphological characterization of Potato (Gopal et al., 2008), Forage sorghum (Joshi et al., 2009), Jute (Begum et al., 2011), Pearl millet (Singh et al., 2016) has been done using DUS guidelines.

In the 31 DUS characters considered for study the genotypes morphologically differed in leaf shape that is Orbicular (Desi Lauki-404, Desi Lauki gol-417, Lauki-426) and Obovate (Lauki-398) (Table 1). Fruit skin colour differed as light green (Desi Lauki-404, Desi Lauki Gol-417, Lauki-426) and Dark green (Desi Lauki-398) (Table 4). Fruit shape the Bottle gourd genotypes have been grouped in to cylindrical (Desi Lauki-404), round (Desi Lauki Gol-417), elongate curved (Desi Lauki398 and Lauki-426) (Table 3). 
Table.1 Pooled morphological characterization of bottle gourd genotypes based on DUS guidelines (kharif-2016 and 2017)

\begin{tabular}{|c|c|c|c|c|c|c|c|}
\hline GENOTYPES & $\begin{array}{l}\text { Plant } \\
\text { growth } \\
\text { habitat } \\
\text { (m) }\end{array}$ & $\begin{array}{l}\text { Stem: } \\
\text { shape }\end{array}$ & $\begin{array}{l}\text { Stem: } \\
\text { pubescence }\end{array}$ & $\begin{array}{l}\text { Stem: } \\
\text { length of } \\
\text { internodes } \\
\text { of main } \\
\text { stem(b/w } \\
15-20 \\
\text { node) cm }\end{array}$ & $\begin{array}{l}\text { Stem: no } \\
\text { of } \\
\text { primary } \\
\text { branches }\end{array}$ & $\begin{array}{l}\text { Leaf } \\
\text { blade: } \\
\text { margin }\end{array}$ & $\begin{array}{l}\text { Leaf: } \\
\text { shape }\end{array}$ \\
\hline $\begin{array}{l}\text { DESI LAUKI- } \\
404\end{array}$ & $\begin{array}{l}\text { Medium } \\
\text { viny }\end{array}$ & Angular & Present & Long & Medium & Serrate & Orbicular \\
\hline $\begin{array}{c}\text { DESI LAUKI- } \\
398\end{array}$ & $\begin{array}{l}\text { Short } \\
\text { viny }\end{array}$ & Angular & Present & Long & Less & Serrate & Obovate \\
\hline $\begin{array}{l}\text { DESI LAUKI } \\
\text { GOL - } 417\end{array}$ & $\begin{array}{l}\text { Long } \\
\text { viny }\end{array}$ & Angular & Present & Medium & Medium & Serrate & Orbicular \\
\hline LAUKI-426 & $\begin{array}{l}\text { Medium } \\
\text { viny }\end{array}$ & Angular & Present & Long & Medium & Serrate & Orbicular \\
\hline
\end{tabular}

Table.2 Pooled morphological characterization of Bottle gourd genotypes based on DUS guidelines (kharif-2016 and 2017)

\begin{tabular}{|c|c|c|c|c|c|c|c|}
\hline CHARACTER & $\begin{array}{l}\text { Leaf: } \\
\text { length } \\
\text { (b/w } \\
15-20 \\
\text { node) } \\
\mathrm{cm}\end{array}$ & $\begin{array}{l}\text { Leaf: } \\
\text { width } \\
\text { (b/w } \\
15-20 \\
\text { node) } \\
\text { cm }\end{array}$ & $\begin{array}{l}\text { Leaf: } \\
\text { pubescence } \\
\text { nature(15- } \\
20 \text { node) }\end{array}$ & $\begin{array}{l}\text { Leaf } \\
\text { blade: } \\
\text { no of } \\
\text { lobes }\end{array}$ & $\begin{array}{l}\text { Tendril: } \\
\text { branching }\end{array}$ & $\begin{array}{l}\text { Petiole: } \\
\text { length } \\
\text { (15-20 } \\
\text { nodes) } \\
\text { cm }\end{array}$ & $\begin{array}{l}\text { Ovary: } \\
\text { length } \\
\text { (on the } \\
\text { day of } \\
\text { anthesis) } \\
\text { cm }\end{array}$ \\
\hline DESI LAUKI-404 & Medium & Medium & Soft & 5 lobes & Branched & Medium & Medium \\
\hline DESI LAUKI-398 & Medium & Medium & Soft & 3 lobes & Branched & Medium & Short \\
\hline $\begin{array}{l}\text { DESI LAUKI } \\
\text { GOL - } 417\end{array}$ & Medium & Broad & Soft & 5 lobes & Branched & Long & Short \\
\hline LAUKI-426 & Medium & Broad & Soft & 5 lobes & Branched & Long & Medium \\
\hline
\end{tabular}


Table.3 Pooled morphological characterization of bottle gourd genotypes based on DUS guidelines (kharif-2016 and 2017)

\begin{tabular}{|c|c|c|c|c|c|c|c|}
\hline GENOTYPES & $\begin{array}{l}\text { Peduncle } \\
\text { length } \\
\text { (cm) }\end{array}$ & $\begin{array}{l}\text { Fruit } \\
\text { :length } \\
\text { (cm) }\end{array}$ & $\begin{array}{l}\text { Fruit: } \\
\text { diameter(cm) }\end{array}$ & $\begin{array}{l}\text { Fruit : } \\
\text { shape in } \\
\text { longitudinal } \\
\text { section }\end{array}$ & $\begin{array}{l}\text { Fruit: } \\
\text { neck }\end{array}$ & $\begin{array}{l}\text { Fruit: } \\
\text { skin } \\
\text { colour }\end{array}$ & $\begin{array}{l}\text { Fruit: } \\
\text { shape of } \\
\text { base at } \\
\text { blossom } \\
\text { end }\end{array}$ \\
\hline DESI LAUKI-404 & Medium & Long & Large & Cylindrical & Straight & $\begin{array}{l}\text { Light } \\
\text { green }\end{array}$ & Semi blunt \\
\hline DESI LAUKI-398 & Medium & Medium & Large & $\begin{array}{l}\text { Elongate } \\
\text { curved }\end{array}$ & Crooked & $\begin{array}{l}\text { Dark } \\
\text { green }\end{array}$ & Blunt \\
\hline $\begin{array}{c}\text { DESI LAUKI } \\
\text { GOL - } 417 \\
\end{array}$ & Medium & Short & Large & Round & Crooked & $\begin{array}{l}\text { Light } \\
\text { green }\end{array}$ & Semi blunt \\
\hline LAUKI-426 & Medium & Long & Large & $\begin{array}{l}\text { Elongate } \\
\text { curved }\end{array}$ & Crooked & $\begin{array}{l}\text { Light } \\
\text { green }\end{array}$ & Blunt \\
\hline
\end{tabular}

Table.4 Pooled morphological characterization of Bottle gourd genotypes based on DUS guidelines (kharif -2016 and 2017)

\begin{tabular}{|c|c|l|l|l|l|}
\hline CHARACTER & $\begin{array}{l}\text { Fruit: } \\
\text { shape of } \\
\text { apex at } \\
\text { peduncle } \\
\text { end }\end{array}$ & $\begin{array}{l}\text { Fruit: } \\
\text { Pubescence }\end{array}$ & $\begin{array}{l}\text { Flesh: } \\
\text { texture }\end{array}$ & $\begin{array}{l}\text { Fruit: } \\
\text { gelatinous } \\
\text { flesh }\end{array}$ & $\begin{array}{l}\text { Seed: } \\
\text { texture at } \\
\text { marketable } \\
\text { stage }\end{array}$ \\
\hline GENOTYPES & Flat & Present & Soft & Present & Soft \\
\hline DESI LAUKJ-404 & Flat & Present & Soft & Present & Soft \\
\hline DESI LAUKJ-398 & Depressed & Present & Soft & Present & Soft \\
\hline DESI LAUKI GOL-417 & Raised & Present & Soft & Present & Soft \\
\hline
\end{tabular}

Table.5 Pooled morphological characterization of Bottle gourd genotypes based on DUS guidelines (kharif-2016 and 2017)

\begin{tabular}{|c|c|c|c|l|l|}
\hline CHARACTER & $\begin{array}{l}\text { Seediness(no of } \\
\text { seeds/fruit at } \\
\text { time of } \\
\text { extraction }\end{array}$ & $\begin{array}{l}\text { Seed: } \\
\text { length }\end{array}$ & $\begin{array}{l}\text { Seed: } \\
\text { width }\end{array}$ & $\begin{array}{l}\text { Seed: } \\
\text { shape }\end{array}$ & $\begin{array}{l}\text { Seed: intensity } \\
\text { of brown colour } \\
\text { of testa }\end{array}$ \\
\hline DESI LAUKJ-404 & High & Large & Large & Rectangular & Light \\
\hline DESI LAUKJ-398 & Medium & Large & Large & Rectangular & Light \\
\hline DESI LAUKI & High & Large & Large & Rectangular & Light \\
\hline GOL-417 & High & Large & Large & Rectangular & Light \\
\hline LAUK-426 & & & & &
\end{tabular}


Table 62016 data for quantitative characters performance of various characteristics in bottle gourd genotypes (kharif-2016)

\begin{tabular}{|c|c|c|c|c|c|c|c|c|c|c|c|c|c|c|}
\hline \multirow[t]{2}{*}{ NO. } & \multirow[t]{2}{*}{ Genotypes } & \multicolumn{13}{|c|}{ Characters } \\
\hline & & $\begin{array}{c}\text { Plant: } \\
\text { growth } \\
\text { habit }\end{array}$ & $\begin{array}{c}\text { Stem: } \\
\text { length of } \\
\text { internodes } \\
\text { of main } \\
\text { stem (b/w } \\
15-20^{\text {th }} \\
\text { node) }\end{array}$ & $\begin{array}{c}\text { Stem: no } \\
\text { of } \\
\text { primary } \\
\text { branches }\end{array}$ & $\begin{array}{c}\text { Leaf: } \\
\text { length } \\
(\text { b/w } 15- \\
2^{\text {th }} \\
\text { node })\end{array}$ & $\begin{array}{c}\text { Leaf: } \\
\text { width } \\
\text { (b/w 15- } \\
20^{\text {th }} \\
\text { node) }\end{array}$ & $\begin{array}{c}\text { Petiole: } \\
\text { length } \\
(\text { b/w } 15- \\
2^{\text {th }} \\
\text { node })\end{array}$ & $\begin{array}{l}\text { Ovary: } \\
\text { length } \\
\text { (on the } \\
\text { day of } \\
\text { anthesis) }\end{array}$ & $\begin{array}{l}\text { Peduncle: } \\
\text { length }\end{array}$ & $\begin{array}{l}\text { Fruit: } \\
\text { length }\end{array}$ & $\begin{array}{c}\text { Fruit: } \\
\text { diameter }\end{array}$ & Seediness & $\begin{array}{l}\text { Seed: } \\
\text { length }\end{array}$ & $\begin{array}{l}\text { Seed: } \\
\text { width }\end{array}$ \\
\hline 1 & $\begin{array}{c}\text { DESI } \\
\text { LAUKI-404 }\end{array}$ & 5.08 & 14.49 & 8.27 & 16.18 & 17.77 & 13.49 & 2.91 & 11.90 & 47.31 & 27.30 & 512.80 & 2.04 & 0.84 \\
\hline 2 & $\begin{array}{c}\text { DESI } \\
\text { LAUKI-398 }\end{array}$ & 3.16 & 13.55 & 5.33 & 19.01 & 18.37 & 12.94 & 2.09 & 10.18 & 28.95 & 25.64 & 327.00 & 1.91 & 0.76 \\
\hline 3 & $\begin{array}{c}\text { DESI } \\
\text { LAUKI GOL } \\
-417\end{array}$ & 5.91 & 12.22 & 7.53 & 15.31 & 22.91 & 16.68 & 2.09 & 13.04 & 18.40 & 35.69 & 413.93 & 1.82 & 0.87 \\
\hline 4 & LAUKI-426 & 4.73 & 15.83 & 7.73 & 17.00 & 24.20 & 17.36 & 2.85 & 10.70 & 67.03 & 26.34 & 451.13 & 1.61 & 0.69 \\
\hline & MEAN & 4.72 & 14.02 & 7.22 & 16.88 & 20.81 & 15.12 & 2.49 & 11.46 & 40.42 & 28.74 & 426.22 & 1.85 & 0.79 \\
\hline & $\mathrm{CV}$ & 5.24 & 5.85 & 7.38 & 5.09 & 5.95 & 5.99 & 6.95 & 5.43 & 5.82 & 8.13 & 5.32 & 5.10 & 5.55 \\
\hline & $\operatorname{Max}$ & 5.91 & 15.83 & 8.27 & 19.01 & 24.20 & 17.36 & 2.91 & 13.04 & 67.03 & 35.69 & 512.80 & 2.04 & 0.87 \\
\hline & Min & 3.16 & 12.22 & 5.33 & 15.31 & 17.77 & 12.94 & 2.09 & 10.18 & 18.40 & 25.64 & 327.00 & 1.61 & 0.69 \\
\hline & SEM & 0.20 & 0.67 & 0.43 & 0.70 & 1.01 & 0.74 & 0.14 & 0.51 & 1.92 & 1.91 & 18.51 & 0.08 & 0.04 \\
\hline & CD@5\% & 0.49 & 1.64 & 1.06 & 1.72 & 2.48 & 1.81 & 0.34 & 1.24 & 4.70 & 4.67 & 45.30 & 0.19 & 0.09 \\
\hline & CD@1\% & 0.75 & 2.48 & 1.61 & 2.60 & 3.75 & 2.74 & 0.52 & 1.88 & 7.12 & 7.07 & 68.63 & 0.29 & 0.13 \\
\hline
\end{tabular}


Table.7 2017 data for quantitative characters Performance of various characteristics in Bottle gourd genotypes kharif (2017)

\begin{tabular}{|c|c|c|c|c|c|c|c|c|c|c|c|c|c|c|}
\hline \multirow[t]{2}{*}{ NO. } & \multirow[t]{2}{*}{ Genotypes } & \multicolumn{13}{|c|}{ Characters } \\
\hline & & $\begin{array}{c}\text { Plant: } \\
\text { growth } \\
\text { habit }\end{array}$ & $\begin{array}{c}\text { Stem: } \\
\text { length of } \\
\text { internodes } \\
\text { of main } \\
\text { stem }(b / w \\
15-20^{\text {th }} \\
\text { node) }\end{array}$ & $\begin{array}{c}\text { Stem: no } \\
\text { of } \\
\text { primary } \\
\text { branches }\end{array}$ & $\begin{array}{c}\text { Leaf: } \\
\text { length } \\
(\mathrm{b} / \mathrm{w} \\
15-20^{\text {th }} \\
\text { node) }\end{array}$ & $\begin{array}{c}\text { Leaf: } \\
\text { width } \\
(b / w \\
15-20^{\text {th }} \\
\text { node) }\end{array}$ & $\begin{array}{c}\text { Petiole: } \\
\text { length } \\
\text { (b/w } \\
15-20^{\text {th }} \\
\text { node) }\end{array}$ & $\begin{array}{l}\text { Ovary: } \\
\text { length } \\
\text { (on the } \\
\text { day of } \\
\text { anthesis) }\end{array}$ & $\begin{array}{l}\text { Peduncle: } \\
\text { length }\end{array}$ & $\begin{array}{l}\text { Fruit: } \\
\text { length }\end{array}$ & $\begin{array}{c}\text { Fruit: } \\
\text { diameter }\end{array}$ & Seediness & $\begin{array}{l}\text { Seed: } \\
\text { length }\end{array}$ & $\begin{array}{l}\text { Seed: } \\
\text { width }\end{array}$ \\
\hline 1 & $\begin{array}{c}\text { DESI } \\
\text { LAUKI-404 }\end{array}$ & 5.13 & 14.45 & 8.13 & 16.17 & 18.29 & 13.79 & 2.77 & 11.85 & 47.85 & 26.53 & 505.73 & 2.03 & 0.80 \\
\hline 2 & $\begin{array}{c}\text { DESI } \\
\text { LAUKI-398 }\end{array}$ & 3.11 & 14.74 & 5.00 & 19.01 & 17.95 & 12.52 & 2.17 & 10.17 & 29.09 & 24.99 & 316.27 & 1.92 & 0.76 \\
\hline 3 & $\begin{array}{c}\text { DESI } \\
\text { LAUKI } \\
\text { GOL - } 417\end{array}$ & 6.24 & 13.42 & 7.67 & 15.41 & 21.81 & 16.83 & 2.74 & 12.70 & 18.09 & 35.61 & 408.60 & 1.81 & 0.89 \\
\hline 4 & LAUKI-426 & 5.03 & 16.77 & 7.73 & 17.09 & 22.97 & 16.57 & 2.87 & 10.84 & 65.22 & 24.67 & 454.53 & 1.63 & 0.69 \\
\hline & MEAN & 4.88 & 14.84 & 7.13 & 16.92 & 20.26 & 14.93 & 2.64 & 11.39 & 40.06 & 27.95 & 421.28 & 1.85 & 0.78 \\
\hline & $\mathrm{CV}$ & 5.22 & 5.08 & 5.70 & 5.03 & 5.10 & 5.17 & 5.36 & 5.30 & 5.11 & 5.14 & 5.55 & 5.15 & 5.72 \\
\hline & $\operatorname{Max}$ & 6.24 & 16.77 & 8.13 & 19.01 & 22.97 & 16.83 & 2.87 & 12.70 & 65.22 & 35.61 & 505.73 & 2.03 & 0.89 \\
\hline & Min & 3.11 & 13.42 & 5.00 & 15.41 & 17.95 & 12.52 & 2.17 & 10.17 & 18.09 & 24.67 & 316.27 & 1.63 & 0.69 \\
\hline & SEM & 0.21 & 0.62 & 0.33 & 0.69 & 0.84 & 0.63 & 0.12 & 0.49 & 1.67 & 1.17 & 19.08 & 0.08 & 0.04 \\
\hline & CD@5\% & 0.51 & 1.51 & 0.81 & 1.70 & 2.06 & 1.54 & 0.28 & 1.21 & 4.09 & 2.87 & 46.70 & 0.19 & 0.09 \\
\hline & CD@1\% & 0.77 & 2.28 & 1.23 & 2.57 & 3.12 & 2.34 & 0.43 & 1.83 & 6.20 & 4.35 & 70.76 & 0.29 & 0.14 \\
\hline
\end{tabular}


Different shapes of fruit, leaf and the colour of the fruit skin clearly indicate the morphological differentiation present among the different genotypes under study. There was no major variation in the individual characters of the respective genotypes during the period of study. The genotypes have been characterized using the DUS guidelines for the two consecutive years. It is concluded that the DUS guidelines can be effectively used for morphological characterization of genotypes which later on helps in the process of testing for DUS of the respective genotypes which can be used for developing new varieties.

\section{Acknowledgements}

First of all I thank Lord Almighty for helping me to successfully complete this research work. I sincerely thank my Advisor Dr. A. K. Chaurasia, co-advisor Dr. Bineeta M. Bara and my dear colleagues Mr. K. Rohan Reddy and Mr. N. Nithyananda for their support all along the research work. I also show my sincere gratitude to all the people who supported me during the research period.

\section{References}

Begum, T., Kumar, D. (2011). Usefulness of morphological characteristics for DUS testing of jute (Corchorus olitorius L. and C. capsularis L.). SPAN J AGRIC RES. 9(2):473-483.

Gopal, J., Pandey, S.K., Kumar, V., Kumar, R., Pande, P.C., Singh, S.V. (2008). Morphological descriptors for DUS testing of potato varieties. PGR Newsletter, FAOBiodiversity. 154: 40-47.

Joshi DC, Shrotria PK, Singh R, Chawla HS (2009). Morphological characterization of forage sorghum [Sorghum bicolor (L.) Moench] varieties for DUS testing. INDIAN J GENET PL BR. 69:383-393.

Miadenovic, E., ognjanov, V., Ljubojevic, Cukanovic, J, Berenji, J. (2012). Genetic variability of bottle gourd and its morphological characterization by multi variate analysis. Arch. Biol. Sci. 64(2): 573853.

Mladenovic, E., Janos, B., Vladislav, O., Marija, K., Mirjana, L. and Jelena, C. (2011). Conservation and morphological characterization of bottle gourd for VII ornamental use. Proceedings. 46th Croatian and 6th International Symposium on Agriculture. Opatija. Croatia (550-553).

PPV \& FR Act. 2001. Protection of Plant Varieties and Farmers Rights Act (No.53 of 2001). Department of Agriculture and \& Cooperation, Ministry of Agriculture, Government of India, Krishi Bhavan, New Delhi.

Singh, S., Yadav, Y. P., Yadav, H. P., Vart, D. and Yadav, N. (2016). Morphological characterization of pearl millet hybrids [Pennisetum glaucum (L.) R. Br.] and their parents. AFR J AGRIC RES., 11(5) 371378.

Sivaraj, N. and Pandravada, S. R. (2005). Morphological Diversity for Fruit Characters in Bottle Gourd Germplasm from Tribal Pockets of Telangana Region of Andhra Pradesh, India. Asian AgriHistory. 9(4): 305-310.

Srivastava V, Mahajan R K, Gangopadhyay K K, Singh M and Dhillon B S. (2001). Minimal Descriptors of Agri-Horticultural CropsPart II. Vegetable Crops. PB Mission Leader National Agricultural Technology Project on Plant Biodiversity (NATPPB) and NBPGR, New Delhi. Monnto Publishing House, NewDelhi.

\section{How to cite this article:}

Gandamala Raghu, Arun Kumar Chaurasia, K. Rohan Reddy, Bineeta M. Bara and Nithyananda, N. 2018. DUS Characterization of Bottle Gourd [Lagenaria siceraria (Mol.) Standl.] Genotypes. Int.J.Curr.Microbiol.App.Sci. 7(10): 680-686. doi: https://doi.org/10.20546/ijemas.2018.710.075 